$17 \%$ had active nephritis, $16 \%$ were in nephritis remission and $67 \%$ had no history of nephritis. Of patients with nephritis, $28 \%$ had membranous class $\mathrm{V}, 27 \%$ had proliferative or proliferative/mixed LN, 3\% class I/II, $0.5 \%$ post-transplant, $0.5 \%$ ESRD on dialysis, and class was unknown in $32 \%$. The prevalence of fibromyalgia was $10.5 \%$ in those with nephritis compared to $23 \%$ of patients without nephritis $(p=0.04)$. Patients with LN had statistically significantly lower fibromyalgia severity scores, symptom severity scores, areas of pain, fatigue, cognitive dysfunction, sleep disturbance, forgetfulness, muscle pain, and numbness. Patient-reported disease activity was significantly lower in patients with LN remission. Depression and anxiety were not significantly different between groups.

Conclusions Patients with both active and inactive lupus nephritis have lower rates of fibromyalgia, fatigue, sleep and cognitive dysfunction compared to non-nephritis lupus patients. Depression and anxiety were pervasive among all lupus groups and persisted even after achieving lower disease activity. The drivers of low HRQoL may be distinct across SLE subgroups. Lupus nephritis patients have high rates of disability and poor HRQoL, however the mechanism underlying these outcomes in nephritis is less likely related to features of fibromyalgia

Funding Source(s): None

\section{INTRODUCTION OF BIOSIMILARS (SIMILAR BIOLOGICAL) AS FINANCIAL SAVING TO OUR COUNTRIES FOR RHEUMATIC DISEASES}

Shereen Abdalfattah*. Benha university Benha faculty of medicine rheumatolgy and rehabilitation department

\subsection{6/lupus-2019-Ism.115}

Background Biosimilars are innovative therapeutic drugs which offer a cheaper alternation for biological which used for management of chronic rheumatic diseases. The aim of this review is to go through the written data to introduce biosimilars in our countries.

Methods Biosimilars are considered as an opportunity for rheumatology patients who are on traditionally expensive biological.

Some countries in (MENA) region Middle East and North Africa are unable to adjust their healthcare expenditure to provide biologic therapies. $71 \%$ rheumatologists from MENA countries at meeting were agreed with European League Against Rheumatism (EULAR) recommendations for introduction of biologic therapy.

Egypt has already established their own regulatory frameworks for biosimilar approval in 2013. Interest in biosimilar researches in MENA region continues grow to introduce price competition, transitioning from biological to biosimilar for rheumatoid, ankylosing, psoriasis and reduce the expenditure of healthcare without any differences in health outcomes which is important from a social perspective.

Results In 2013 the European Medicines Agency (EMA) has approved biosimilar for treatment of inflammatory disease. Adalimumab biosimilars will be available in the European market at the end of 2018. Switching to rituximab biosimilars in rheumatic conditions is still restricted to small sized studies with limited reporting of efficacy and safety outcomes. Depend on evidence several biosimilars approved by the EMA for adalimumab (BI 695501, SB5, and ABP 501), infliximab (SB2, CT-P13, and infliximabqbtx), etanercept (GP2015 and SB4) and rituximab (CTP10 and GP2013). The duration of treatment were detected in many trials Published data in EMA Public Assessment Reports (EPAR), PubMed and European League Against Rheumatism (EULAR) and American College of Rheumatology (ACR).

Conclusions Switching to biosimilars in the treatment of inflammatory rheumatic conditions continue as great deal of attention give promise of significant cost saving. Biosimilars could be a good option as first line therapy based on available data but clinical studies are still not sufficient.

Funding Source(s): None

\section{EARLY LIFE BODY SIZE AND RISK OF SYSTEMIC LUPUS ERYTHEMATOSUS}

${ }^{1}$ Soren Jacobsen*, ${ }^{2}$ Peter Engel Thomas, ${ }^{3}$ Julie Aarestrup, ${ }^{3}$ Kathrine Kold Sørensen, ${ }^{3}$ Britt Wang Jensen, ${ }^{3}$ Jennifer Lyn Baker. 'Lupus and Vasculitis Clinic, Rheumatology, Copenhagen University Hospital, Rigshospitalet; ${ }^{2}$ Center for Clinical Research and Prevention, Bispebjerg and Frederiksberg Hospital; ${ }^{3}$ Center for Clinical Research and Prevention, Bispebjerg and Frederiksberg Hospital, Denmark

\subsection{6/lupus-2019-Ism.116}

Background Systemic lupus erythematosus (SLE) is a chronic autoimmune disease. Adult obesity may increase risks of SLE. SLE autoantibodies have been detected in patients many years prior to a SLE diagnosis, suggesting that etiological factors may influence SLE risks early in life. Child or adult height as a risk factors for SLE has not been investigated. However, there are suggestions of a genetic link between SLE and tall adult height. The evidence of an association between birth weight and SLE risks is inconsistent. We therefore investigated whether birth weight, childhood body mass index (BMI $\left.\left[\mathrm{kg} / \mathrm{m}^{2}\right]\right)$, and height are associated with later risks of SLE.

Methods We used the Copenhagen School Health Records Register which contains annual weight and height measurements at ages 7 to 13 years on 406308 children born from 1930-1996 who attended schools in Copenhagen. Information on birth weight was obtained from 1936 onwards. SLE diagnosis was obtained through linkage to the Danish National Patient Register using unique personal identification numbers. Cox hazard regressions were performed to estimate hazard ratios (HR) and 95\% confidence intervals (CI).

Results 346545 children (1 75494 boys) were included. During 40 years of follow-up there were 435 cases of SLE (69 men). As there were no significant interactions with sex, analyses are presented for sex combined. For birth weight there was no significant association with SLE. For childhood BMI there was a positive and significant, or borderline significant, association with SLE at all childhood ages. At age 13 years the HR was 1.13 (95\% CI: 1.02-1.26) per BMI z-score and HRs were similar across all ages. For childhood height there were significant and positive associations with SLE at all 
childhood ages. At age 13 years the HR was 1.12 (95\% CI: 1.01-1.23) per height $\mathrm{z}$-score and HRs were similar across all ages.

Conclusions These findings of positive associations between childhood BMI and height and SLE risk suggest that early life factors may be important in the etiology of SLE.

Funding Source(s): University of Copenhagen Fund for Medical Students and University of Copenhagen, Faculty of Health and Medical Sciences Fund, Gigtforeningen.

\section{ACUTE INFLAMMATORY DEMYELINATING POLYNEUROPATHY AS A PRIMARY MANIFESTATION OF LUPUS IN A PREGNANT PATIENT A RARE AND AN ATYPICAL PRESENTATION}

${ }^{1}$ Anam Umar*, ${ }^{1}$ Muhammad Bilal, ${ }^{2}$ Bina Umar, ${ }^{1}$ Eric Chang, ${ }^{1}$ Balsam Elhammali, ${ }^{1}$ Cinnamon Bradley. ${ }^{1}$ Morehouse School of Medicine; ${ }^{2}$ Henry Ford hospital

\subsection{6/lupus-2019-|sm.117}

Background Systemic lupus erythematosus (SLE) is associated with several neuropsychiatric syndromes involving both the central and peripheral nervous systems. These syndromes are related with SLE progression and thus, are rarely present at the onset of disease. We are reporting a rare case of acute inflammatory demyelinating polyneuropathy (AIDP) as the first manifestation of SLE in a previously asymptomatic patient.

Methods Not applicable as it is a clinical vignette

The abstract for clinical vignette is uploaded as a separate document

Results Not applicable as it is a clinical vignette

The abstract for clinical vignette is uploaded as a separate document

Conclusions Symptoms of AIDP frequently start in lower extremities, yet in about $10 \%$ of patients, arm or facial muscle weakness can be the initial presentation. CSF analysis typically revealed an elevated protein with a normal white cell count; however, a normal CSF protein is observed in onethird to one-half of patients when tested early in the disease course. Our patient had upper extremity weakness as well as initial negative CSF findings, which led to the delay in diagnosis. It is imperative to have a sound knowledge of the typical as well as atypical neurological manifestations of SLE to reduce the morbidity and mortality rate of patients.

Funding Source(s): None

\section{GRANZYME B PRODUCING B CELLS IN SYSTEMIC LUPUS ERYTHEMATOSUS PATIENTS AND LUPUS-LIKE MOUSE MODELS}

Mingxin Bai*, Yin Su. Department of Rheumatology and Immunology Peking University People's Hospital

\subsection{6/lupus-2019-Ism.118}

Background Systemic lupus erythematosus (SLE) is a chronic and systemic autoimmune disease, which is accompanied by abnormal activation of $\mathrm{T} / \mathrm{B}$ lymphocytes, multiple autoantibodies and immune complex deposition. Granzyme B producing $(\mathrm{GrB}+)$ B cells are a group of new regulatory $B$ cell subsets, which can participate in the pathogenesis of autoimmune disease, but its role in SLE is not clear. In this study, we investigate the expression level of Granzyme B producing B cells in SLE patients and lupus-like mouse models.

Methods 1. Patients with SLE $(n=7)$ meeting 1997 American College of Rheumatology revised criteria were enrolled from the Department of Rheumatology and Immunology. 15 healthy individual samples were obtained from physical examination center in our hospital. We collected $4 \mathrm{~mL}$ peripheral anticoagulant blood from both groups and peripheral blood mononuclear cells were isolated. The proportion of $\mathrm{GrB}+\mathrm{B}$ cells in PBMC was identified by flow cytometry. 2. A lupus-like mouse model induced by transfer of spleen cells from bm12 mice was constructed. Two wildtype C57BL/c mice and two lupus-like mouse models (both 68 weeks old mice) were sacrificed by $\mathrm{CO}_{2}$ anesthesia. The spleens of the mice were aseptically isolated, and the spleen single cells were obtained after grinding the tissues. The ratio of $\mathrm{GrB}+$ Breg cells in the spleen cells of the two groups was detected by flow cytometry (FACS). 3. SPSS24.0 software was used for statistical analysis, and $\mathrm{p}<0.05$ was considered statistically significant

Results 1 . Using flow cytometry 7AAD label excludes dead cells, CD3 marker excludes $\mathrm{T}$ lymphocytes and CD56 markers exclude NK cells. After CD14 labeling excludes monocytes/macrophages, the proportion of $\mathrm{GrB}+\mathrm{Breg}$ cells in peripheral blood of healthy controls was 7.52\%; Compared with healthy controls, the proportion of GrB + Breg cells in peripheral blood of SLE patients (4.45\%) showed a significant downward trend. 2. Compared with the wild type control mice, the proportion of $\mathrm{GrB}+$ Breg cells in the spleen cells of lupus mouse model (2.14\%) showed a significant downward trend.

Conclusions The proportion of $\mathrm{GrB}+$ Breg cells in the peripheral blood of SLE patients and the spleen cells of lupus-like mouse models showed a downward trend, which may be related to the pathogenesis of SLE, but the exact role remains to be further verified.

Funding Source(s): This study was supported by grants from the National Natural Science Foundation of China.

\section{NO-SYNTHASE INDUCTIBLE-2 (NOS2) AND VASCULAR ENDOTHELIAL GROWTH FACTOR (VEGF) POLYMORPHISMS IN SYSTEMIC LUPUS ERYTHEMATOSUS AMONG ALGERIAN PATIENTS}

${ }^{1}$ Mounira Benidir*, ${ }^{2}$ Sofiane Samir Salah, ${ }^{3}$ Nesrine Benrebha, ${ }^{4}$ Malik Djennane, ${ }^{5}$ Hachemi Djoudi, ${ }^{6}$ Habiba Amroun, ${ }^{7}$ Ryad Tamouza, ${ }^{8}$ Nabila Attal. ${ }^{1}$ Department of Immunonology, Pasteur Institute of Algeria; ${ }^{2}$ Immunology Department, Mustapha Pacha Hospital; ${ }^{3}$ Medical Biology Department, Blida Hospital; ${ }^{4}$ Rheumatology Department, TiziOuzou Hospital; ${ }^{5}$ Rheumatology Department, Douera Hospital; ${ }^{6}$ Medical Biology Department, Hussein Dey Hospital; ${ }^{7}$ Department of Immunology and Immunogenetics, Henri Mondor Hospital, Paris. France; ${ }^{8}$ Immunology Department, Pasteur Institute of Algeria

\subsection{6/lupus-2019-Ism.119}

Background The development of Systemic Lupus Erythematosus (SLE) depends inter alia on genetic factors including genes involved in oxidative stress and angiogenesis as NOS2 and VEGF. The aim of our study is to evaluate the Single Nucleotide Polymorphisms (SNPs) influence of NOS2 gene (rs2779248, rs2779251 and rs8078340) and VEGF gene (rs1570360 and rs2010963) on SLE development in Algerian patients.

Methods This is a case-control study of 157 SLE patients (age: $37 \pm 2$ years, sex ratio: 1: 10, disease duration: $7.6 \pm 4.3$ years, SLEDAI: $7.3 \pm 6.1$ ) and 173 healthy controls (age: $28 \pm 9$ years, sex ratio: 1: 7). We performed NOS2 and VEGF genes 